\title{
Antikoagulanzien reduzieren nicht den Schmerz bei Patienten mit distaler TVT
}

Righini M et al. Effect of anticoagulant treatment on pain in distal deep vein thrombosis: an ancillary analysis from the cactus trial. J Thromb Haemost 2019; 17 : 507-510

Die isolierte tiefe Venenthrombose (TVT) der Wade, auch als distale TVT bezeichnet, ist häufig und repräsentiert bis zu $50 \%$ aller TVT der unteren Extremität. Das optimale Management dieser Erkrankung bleibt kontrovers. Im vor kurzem publizierten CACTUSTrial war die Behandlung mit Vollantikoagulation der Placebo-Behandlung nicht überlegen in Hinblick auf die Reduktion des Risikos für proximale Ausdehnung oder thrombembolische Ereignisse nach distaler TVT, war jedoch mit signifikant höherem Risiko der Blutung vergesellschaftet.
Rhigini und Kollegen aus Genf untersuchten in ihrer Nebenanalyse des CACTUSTrials, ob niedermolekulare Heparine den Schmerz bei Patienten mit distaler TVT reduzieren können. Dafür schlossen sie 252 Patienten in eine klinische Placebokontrollierte Multicenterstudie ein. Der Schmerz wurde mittels visueller AnalogSkala (VAS) bei jeder Visite registriert.

\section{Ergebnisse}

130 Patienten wurden in den therapeutischen Nadroparin-Arm randomisiert, 122 in den Placebo-Arm. Das mittlere VAS-Ni- veau lag bei Einschluss bei 4,6 (Standardabweichung 2,5), bei 2,1 (Standardabweichung 2,0) nach 1 Woche und bei 0,4 (Standardabweichung 1,2) nach 6 Wochen. Es zeigte sich kein Unterschied in der mittleren VAS-Reduktion zwischen Nadroparin- $(n=106)$ und Placebo-Arm $(n=109)$ nach 1 Woche und nach 6 Wochen. Kompressionsstrümpfe dagegen waren mit einer Reduktion des Schmerzes assoziiert. 
FAZIT

Der CACTUS-Trial zeigte keinen klaren Benefit für die Antikoagulation im Vergleich zu Placebo bei Patienten mit distaler tiefer Venenthrombose in Hinblick auf thrombembolische Komplikationen versus Blutung. Die von den Autoren durchgeführte Nebenanalyse des CACTUS-Trials zeigte darüber hinaus, dass der Gebrauch von niedermolekularen Heparinen nicht die Schmerzkontrolle verbessert im Vergleich zu Placebo-behandelten Patienten mit akuter distaler TVT. Die Autoren der Arbeit kommen zu dem Schluss, dass weitere Studien notwendig sind, um das optimale Management von Patienten mit distaler tiefer TVT zu definieren.

MOR Dr. med. Benedikt Lampl, Regensburg 